\title{
Los efectos de la enseñanza global del periodismo: Análisis de la influencia anglosajona en el aprendizaje de las nuevas tecnologías informativas ${ }^{1}$
}

\author{
María Bella PALOMO TORRES \\ Universidad de Málaga \\ bellapalomo@uma.es
}

Recibido: $17 / 07 / 2012$

Aceptado: 12/10/2012

\section{Resumen}

Muchas de las asignaturas de los estudios de Periodismo requieren una renovación constante para adaptar sus contenidos a contextos actuales que, debido a la influencia tecnológica, son muy cambiantes. En la era de la sociedad de la información y en el marco del Espacio Europeo de Educación Superior, la consulta de los programas implantados en otras universidades se ha convertido en una práctica habitual que permite la orientación de los profesores noveles, la actualización de programas y una ampliación de los recursos disponibles para lanzar una oferta académica global más competitiva. En el presente artículo se exponen los efectos derivados de la consulta y el uso de recursos de prestigio extranjeros en tres asignaturas de los estudios de periodismo en la Universidad de Málaga.

Palabras clave: recursos, TIC, docencia bilingüe, periodismo, multimedia

\section{The effects of global journalism education: Analysis of Anglo influence in the learning of new information technologies}

\begin{abstract}
Many of the Journalism studies subjects require constant renewal to adapt their content to current contexts, due to the influence of technology, are very changeable. In the era of the information society and within the European Higher Education Area, the consultation of the programs implemented in other universities has become a common practice that allows the orientation of new professors, updating programs and an expansion of available resources to launch a more competitive global academic offerings. This paper presents the effects of the consultation and the use of prestigious foreign resources in three subjects of Journalism studies at the University of Málaga.
\end{abstract}

Keywords: resources, ICT, bilingual teaching, journalism, multimedia

\section{Referencia normalizada}

PALOMO TORRES, María Bella (2012): "Los efectos de la enseñanza global del periodismo: Análisis de la influencia anglosajona en el aprendizaje de las nuevas tecnologías informativas". Estudios sobre el mensaje periodístico. Vol. 18, núm. especial noviembre, págs.: 681-689. Madrid, Servicio de Publicaciones de la Universidad Complutense.

Sumario: 1. Introducción. 2. Metodología. 3. Resultados; 3.1. La docencia bilingüe; 3.2. Recursos empleados; 3.2.1. Webs/blogs personales de periodistas y profesores de Periodismo norteamericanos; 3.2.2. Cursos on line y tutoriales de universidades norteamericanas; 3.2.3. Publicaciones especializadas y tutoriales procedentes de webs privadas; 3.2.4. Recursos de medios de comunicación; 3.3. La expansión de la docencia práctica; 3.4. Impulso del trabajo cooperativo; 3.5. La visibilidad del trabajo del estudiante. 4. Conclusiones. 5. Referencias bibliográficas

1 Este trabajo forma parte del proyecto "Audiencias Activas y Periodismo: Estrategias de Innovación en la Empresa Informativa y Nuevas Figuras Profesionales” (CSO2012-39518C04-04). 


\section{Introducción}

La tendencia a unificar el espacio de educación superior y a convertir las tecnologías de la información y la comunicación en la columna vertebral del proceso enseñanzaaprendizaje ha revolucionado la cultura universitaria. Esta apertura genera un modelo de educación transfronterizo, más permeable a las interacciones internacionales.

En este escenario resulta obligatoria una mirada al mundo anglosajón. Las universidades norteamericanas, con una tradición más dilatada en el tiempo en la exposición pública en internet de programas, recursos, cronogramas, actividades y sistemas de evaluación, han servido de inspiración para aquellos centros donde se ha implantado por primera vez la enseñanza de los nuevos medios (Palomo, 2008: 183-195). Además de facilitar el reciclaje del docente español, las universidades norteamericanas han impulsado el aprendizaje bilingüe en nuestras aulas al ofertar cursos online y tutoriales gratuitos.

En esta aldea global del conocimiento, algunos docentes han defendido una mayor formación internacional del alumnado, lo que contribuye a mejorar la enseñanzaaprendizaje de idiomas y de las propias disciplinas (Cuadrado, Ruiz Molina y Coca, 2009: 505-518). Concretamente desde el ámbito periodístico, María Jesús Casals (2006) defiende un modelo de globalización que genere la necesidad de una comunicación intercultural, de diálogo y debate, de conocimiento del mundo.

\section{Metodología}

Por acuerdo del Consejo de Gobierno de la Universidad de Málaga del 21 de julio de 2011, los alumnos que se encuentren matriculados en dicha universidad en alguna de las titulaciones oficiales de grado deberán acreditar, antes de la expedición del título académico, el conocimiento de un segundo idioma con un nivel mínimo correspondiente al B1 del Marco Común Europeo de Referencia para las Lenguas. Esta exigencia justifica el presente estudio, cuyo objetivo general marcado ha sido conocer el impacto real que tiene el inglés en los estudios de Periodismo de la UMA. Basado en el método del caso, se ha realizado un estudio descriptivo de las distintas metodologías docentes implantadas en tres asignaturas de carácter tecnológico (Tecnología de los Medios Escritos; Periodismo Interactivo y Creación de Medios Digitales; Edición Digital) para exponer la permeabilidad que presentan hacia los contenidos anglosajones. Para medir los efectos del uso de este tipo de material, se ha monitorizado los documentos consultados por los alumnos de Periodismo Interactivo en el campus virtual durante el curso 2011/2012, ya que en esta asignatura es donde se realizó una aplicación más intensiva del inglés.

\section{Resultados}

A continuación se desarrolla lo que, tras la experiencia analizada, consideramos principales efectos de este flujo global de enseñanza-aprendizaje, de marcada influencia anglosajona: el incremento de la oferta de enseñanza bilingüe y el acceso a recursos en inglés; la revitalización de la docencia práctica y la apuesta por la exposición pública de los proyectos de estudiantes. 


\subsection{La docencia bilingüe}

Tanto en el ámbito privado como en el público, en España existen apuestas decididas por la enseñanza bilingüe, modalidad contemplada en los grados de Periodismo de la Universidad CEU San Pablo de Madrid y la Universidad Carlos III, o en el título propio International Media Program de la Universidad de Navarra.

En la licenciatura de Periodismo de la Universidad de Málaga se ofertó de forma pionera y aislada en el curso 2007-2008 la asignatura optativa de segundo ciclo Periodismo Interactivo y Creación de Medios Digitales en inglés. La reducción del número de matriculados en un 75 por ciento desaconsejó la continuidad de la experiencia en años posteriores. A pesar de esta resistencia, desde ese curso se ha intentado incrementar el protagonismo de la lengua inglesa en los estudios de Periodismo de la Universidad de Málaga y, especialmente, en las asignaturas de marcado carácter tecnológico. Para que el alumno esté a la vanguardia de los nuevos avances y fomentar el aprendizaje continuo y autónomo, los docentes utilizan material especializado elaborado por universidades extranjeras y publicaciones de prestigio internacional, y parte de ellos quedan a disposición del alumno en el campus virtual. Actualmente cada asignatura pone a su disposición una media de 38 recursos en inglés, lo que significa que el $54 \%$ del material con el que trabaja el alumno está en dicho idioma.

\subsection{Recursos empleados}

En 2006, solo el 36 por ciento de las referencias bibliográficas empleadas en las 17 asignaturas que impartían periodismo digital en España eran recursos digitales (Tejedor, 2008: 33). Esta cifra se considera reducida ya que los recursos on line fortalecen la enseñanza del ciberperiodismo, al incluir las últimas recomendaciones del sector. Tampoco se debe ignorar el potencial del material audiovisual alojado en espacios sociales como Youtube, donde se localizan vídeos en los que explican, por ejemplo, la historia de la imprenta, cómo funcionaban las redacciones durante la era mecánica con linotipias y monotipias, cómo se fabrica el papel, ejemplos de e-paper, la revolución de los medios sociales o cómo construir la portada de una revista con InDesign o Photoshop.

Al margen de este tipo de espacios generalistas, con objeto de fomentar el autoaprendizaje, la capacidad reflexiva y de investigación del alumno, en la experiencia aquí presentada se ha trabajado fundamentalmente con cuatro tipos de materiales en inglés: webs/blogs personales de periodistas y profesores de Periodismo norteamericanos; cursos on line y tutoriales de universidades norteamericanas; publicaciones especializadas y tutoriales procedentes de webs privadas, y recursos de medios de comunicación. La combinación de estos cuatro ejes ha permitido que el alumno sea consciente de la demanda real del mercado.

A continuación se detalla el material utilizado dentro de los cuatro bloques aludidos:

\subsubsection{Webs/blogs personales de periodistas y profesores de Periodismo nortea- mericanos}

Los espacios elaborados por profesores han resultado de especial interés para el docente, al visualizar otras estrategias contemporáneas para abordar asignaturas tecno- 
lógicas. El uso más intensivo se ha realizado en el sitio de Mindy McAdams, profesora de la Universidad de Florida que posee varias webs dedicadas al periodismo on line desde las que se puede acceder a actividades relacionadas con su libro Flash Journalism (2004), y también a la metodología y los recursos que aplica en sus asignaturas. Otras webs destacables han sido la del profesor de la Universidad de Columbia, Sree Sreenivasan; David Carlson, profesor de nuevos medios en la Universidad de Florida, y Alfred Hermida, de la University of British Columbia. Esta documentación se complementó con los recursos publicados por la Universidad de Annenberg para la enseñanza del periodismo digital.

Respecto a los periodistas, se tomaron como ejemplo algunas webs personales para que sirvieran de modelo a los alumnos que en el marco del desarrollo de su identidad digital, construyeron su propio sitio y desearon dotarlo de un sello profesional.

\subsubsection{Cursos on line y tutoriales de universidades norteamericanas}

Los dos sitios webs más empleados en nuestra experiencia (News University y Knight Digital Media Center) tienen un nexo común: son financiados por la Fundación Knight Ridder. News University, del Poynter Institute, es uno de los espacios más fértiles para que tanto docentes como alumnos actualicen conocimientos en el campo periodístico. Fundada en 2005, cuenta con más de 215.000 usuarios registrados, de los que un $15 \%$ proceden de fuera de Estados Unidos. A través de su web se puede acceder a más de 250 cursos gratuitos o con el sello de low-cost, que en unos casos son interactivos y en otros, vídeos. Las temáticas abordan desde cómo ser un periodista emprendedor a dominar técnicas del reportaje medioambiental, pasando por la interpretación de encuestas o cómo aplicar el color en prensa.

Una quinta parte de las prácticas realizadas en las asignaturas de ciberperiodismo en España son ejercicios dedicados a la creación de materiales multimedia informativos, donde el alumno adapta la estructura de estos mensajes a las posibilidades que introduce internet: interactividad, hipertextualidad, multimedialidad... (Tejedor, 2008: 32). La exigencia de este tipo de contenidos influyó en la elección del Knight Digital Media Center como segundo recurso para completar la dimensión instrumental de la asignatura Periodismo Interactivo y Creación de Medios Digitales. Desarrollado por la Escuela de Periodismo de la Universidad de Berkeley, aloja en su web tutoriales de acceso abierto sobre audio, visualización de datos, Flash, mapas, fotografía, bases de datos, reportajes, medios sociales, vídeo y desarrollo web.

Esta vertiente técnica se complementa con la visualización de decenas conferencias que distintos expertos han impartido en Berkeley y se encuentran alojadas en Vimeo. Las temáticas son fundamentalmente el futuro de los medios, la innovación, los medios sociales, el diseño y la redacción periodística.

\subsubsection{Publicaciones especializadas y tutoriales procedentes de webs privadas} Editor\&Publisher, Cyberjournalist.net, SocialTimes, Social Media Examiner, 1000 Word: Where Journalism and Technology Meet, Journalism.org y Multimedia Learning constituyen las principales fuentes de actualidad de las que se nutren las materias de carácter tecnológico analizadas para presentar novedades y provocar debates en el aula. 
La mayoría de los recursos aquí expuestos son de uso voluntario para los alumnos, pero sí existen otras webs de acceso casi obligado para poder superar las materias, y son aquellas que albergan maquetas y tutoriales que necesitan los estudiantes para perfeccionar sus conocimientos técnicos y elaborar sus publicaciones digitales. Dentro de este grupo se encuentran un videotutorial del programa de autoedición profesional QuarkXPress 8, Art for the Web, Get Free Templates, Template Basics, DynamicDrive, Entheos, Speckyboy Design Magazine, Color Palette Generator, Color Hunter y los editores de menú Izzymenu y Button Generator.

\subsubsection{Recursos de medios de comunicación}

En el marco europeo se ha tomado como referencia la propuesta formativa on line para periodistas que posee la BBC desde 2003, a través de su división Training \& Development. Creado con objeto de extender los estándares de calidad, los recursos y la experiencia de la $\mathrm{BBC}$ a periodistas de todo el mundo, esta oferta dejó de ser abierta en 2011 y se ha transformado en la BBC Academy. Aquí se enmarca el BBC College of Journalism, a cuyos cursos on line solo se puede acceder si el ordenador que utilizamos está dentro del Reino Unido ya que se ha transformado en un servicio dirigido a aquellos ciudadanos que pagan el canon de la televisión británica. Oxford University Press comercializa las suscripciones externas.

A pesar de esta limitación en el acceso a cursos, se continua recomendando en las asignaturas la lectura de textos en abierto, como las normas de estilo visual para el contenido web, la política de uso de redes sociales, microblogs y otras webs de terceras partes en la BBC o la normativa sobre cómo interactuar con las audiencias.

Estas visiones se completan con visitas a las secciones de reportajes multimedia e infografías interactivas de medios como The New York Times o USA Today, donde han publicado un recopilatorio de opciones para construir reportajes multimedia que sirven de inspiración para los proyectos que deben desarrollar los alumnos. Tampoco se puede olvidar el Laboratorio de I+D que posee The New York Times, ya que a través de su web se pueden visionar en el aula vídeos que anticipan lo que puede ser el futuro del consumo de medios, o la sección Open Journalism, del diario The Guardian, dedicada a redefinir la visión tradicional del periodismo para otorgar un rol más activo a las audiencias.

Tras monitorizar la consulta que se realizó de todo este tipo de material en lengua inglesa en la asignatura Periodismo Interactivo y Creación de Medios Digitales, se observó que la totalidad de los alumnos accedieron a los tutoriales, las maquetas, los efectos y recursos en inglés para poder ejecutar sus proyectos. Sin embargo, el número de estudiantes que con carácter voluntario leyó total o parcialmente artículos o libros recomendados en inglés de disposición libre en internet, como We Media, Mediactive o The Cluetrain Manifesto, se redujo al 43,1\%.

\subsection{La expansión de la docencia práctica}

Más allá del dominio de un nuevo idioma, la internalización del conocimiento permite asumir nuevas estrategias de aprendizaje que capaciten al alumno para asumir un rol más competitivo en la sociedad global. 
Uno de los ejes sobre los que se sustenta el Plan Bolonia, consiste en superar el tradicional sistema memorístico para primar el raciocinio y la capacidad de trabajo. En el proceso de adaptación a este nuevo contexto y para modernizar y hacer más funcional la estructura de las asignaturas, la dimensión práctica adquiere un mayor relieve. Por este motivo, los ejercicios prácticos propuestos en las asignaturas de carácter tecnológico en los estudios de Periodismo de la Universidad de Málaga simulan actividades propias del ejercicio profesional, y pueden ser realizados en inglés o español, a voluntad del estudiante. Como ejemplo de esta práctica, un 10\% de las portadas y reportajes que crearon para Diseño Digital fueron realizadas en lengua inglesa.

Pero esta aproximación entre la academia y la empresa no es una novedad en el entorno universitario, ya que se aplica en Estados Unidos desde hace más de un siglo: "Si se analizan los programas de las escuelas de periodismo desde 1908 a 1930 se comprueba la preponderancia de las clases prácticas" (Gordón Pérez, 1991: 51).

El día que comenzaron las clases en la Escuela de Periodismo de la Universidad de Missouri (Estados Unidos), considerada la primera escuela de periodismo del mundo al ser fundada el 14 de septiembre de 1908 (Winflield, 2008), los alumnos prepararon el primer número del periódico University Missourian, que en la actualidad sigue funcionando bajo el título Columbia Missourian. Además, en la Escuela de Periodismo de la Universidad de Missouri es una tradición que los estudiantes matriculados en el curso The Community Newspaper realicen una estancia de una semana en periódicos locales y participen de su elaboración para incrementar su conocimiento desarrollando cada día distintas funciones. Esta práctica, basada en el lema 'learning by doing', la introdujo el primer decano de la facultad, Walter Williams, a finales de los años 20 .

Estos ejercicios de simulación pueden tener carácter individual o colectivo, destacando dentro de este campo los de carácter cooperativo (Carratalá, 2011: 21-40).

\subsection{Impulso del trabajo cooperativo}

Conforme avanza la tecnología, el modelo clásico de enseñanza-aprendizaje, basado en la comunicación lineal entre profesor y alumnos, deja paso a otros que, mediante el uso de aulas virtuales y el apoyo de espacios como Facebook (García, 2008: 49-59), Myspace o Twitter posibilitan una interacción y comunicación en red bidireccional.

El campus virtual de la Universidad de Málaga se ha convertido en la columna vertebral de la enseñanza en dicha institución. Durante el curso 2011-2012 en este espacio se alojaron cuarenta y siete asignaturas vinculadas a los estudios de Periodismo ( 1 . $^{\circ}$ y $2 .^{\circ}$ de grado; $3 .{ }^{\circ}$ y $4 .^{\circ}$ de licenciatura), lo que genera una intensa actividad on line tanto por parte de profesores como alumnos. Además de mejorar la comunicación entre estos dos actores, este tipo de plataforma permite aproximar al alumno de Periodismo a su futuro entorno de trabajo, marcado por la virtualidad y el trabajo en red. La relación del redactor con las agencias, con los corresponsales y con las fuentes es fundamentalmente no presencial, y se han incrementado el número de reuniones y ruedas de prensa basadas en la videoconferencia (Machill, Beiler y Schmutz, 2006: 869-888).

En el caso de la asignatura Periodismo Interactivo, durante el curso los alumnos crean blogs, webs personales, introducciones en Flash, imágenes de $360^{\circ}$, ejercicios 
de geolocalización, corrigen textos online, diseñan webs, descubren el CSS y el último mes se aprovecha esa inteligencia colectiva para que trabajen en grupo de forma cooperativa realizando reportajes multimedia, en los que ellos se responsabilizan de seleccionar el tema, documentarse, diseñar el storyboard, realizar la cobertura, grabar y editar en vídeo/audio, hacer la fotografía, la redacción y el montaje de la web. En definitiva, se transforman en periodistas multimedia. El éxito de este proyecto reside en la existencia de diálogo, de negociación, de autoevaluación y el respeto. Para lograrlo, previamente se realiza un estudio de las capacidades, deficiencias y posibilidades de los alumnos, y en función de ello se componen los grupos, para que sean equilibrados. Como resultado, los alumnos se sienten autosuficientes pero no aislados y comprenden el significado de la responsabilidad compartida.

En otras asignaturas como Tecnología de los Medios Escritos o Diseño Digital se aproxima al estudiante a la cultura de la participación construyendo en el campus virtual espacios donde publicar regularmente sus trabajos, así como revisar y criticar los del resto para que puedan aprender de sus mejoras y sus errores.

También en Tecnología de los Medios Escritos han elaborado wikis colectivas donde recopilaban semanalmente los apuntes tomados en las clases prácticas, de forma que el alumno se transforma en autor de su propio manual práctico de la asignatura, lo que ha permitido al profesorado comprobar secuencialmente si las explicaciones eran comprendidas correctamente.

\subsection{La visibilidad del trabajo del estudiante}

Además de adquirir un conocimiento transnacional de la profesión, en este entorno el alcance de las actividades del alumno también ha sufrido una transformación, y nacen nuevas plataformas en las que difundir sus avances, más allá del campus virtual. En la web de la Escuela de Periodismo de la Universidad de Columbia hay una sección denominada Student Work Online, donde se almacenan las publicaciones digitales que han elaborado los alumnos para que cualquier navegante de la red explore su labor. En esta misma línea han creado espacios la City de Londres, la Universidad de Minnesota o la American University.

Partiendo de la hipótesis de que esta exposición pública del trabajo del estudiante incrementa la responsabilidad, la autocrítica y el perfeccionismo del alumno, se decidió que en la web del Departamento de Periodismo de la Universidad de Málaga también quedaran expuestos los mejores reportajes multimedia de los alumnos, dando visibilidad a un esfuerzo que la mayoría de las veces queda almacenado en el cajón de un profesor.

En el caso de Diseño Digital, sus portadas y reportajes de revistas protagonizaron en mayo de 2012 la exposición "Diamantes en bruto" en la Galería Central de la Facultad de Ciencias de la Comunicación. Otros mecanismos que existen para promocionar el trabajo del estudiante proceden de los medios sociales. En Scribb o Issuu los docentes también publican una recopilación de los proyectos realizados por los alumnos en Diseño Digital.

En definitiva, se trata no solo de tutorizar u orientar al alumno; el docente también se puede convertir en promotor de su talento. 


\section{Conclusiones}

Aunque durante los últimos años de vida de las licenciaturas, la enseñanza las nuevas formas del periodismo resultaba preocupante (Tejedor, 2008: 30), el renacimiento del sistema educativo bajo la denominación Espacio Europeo de Educación Superior aporta esperanza a la reinvención de dichas clases al existir un amplio abanico de recursos que potencian el enfoque teórico-práctico de las asignaturas tecnológicas. Este sistema de aprendizaje permite al estudiante transformarse en un sujeto proactivo capaz de contextualizar su conocimiento y asimilar nuevas vías de formación continua.

En este artículo se realiza una apuesta decidida por combinar la innovación tecnológica y la innovación pedagógica, por ofertar conocimientos útiles y actualizados que respondan al contexto periodístico actual, primando el aprendizaje horizontal, donde el alumno está influido no solo por el profesor, sino también por agentes externos e internos, que incrementan la autocrítica, la implicación, la motivación y el compromiso del alumno, capaz de generar nuevas ideas y productos.

Según los datos expuestos de la experiencia, la influencia de la academia anglosajona en el aprendizaje del alumno queda asegurada de una forma directa solo cuando el docente asume el rol de traductor insertando en su discurso dichas lecturas, o cuando se evalúa el uso de materiales en otro idioma, ya que el alumno solo consulta en inglés aquella documentación necesaria para ejecutar los proyectos prácticos de los que depende para superar la asignatura, y menos de la mitad invierte su tiempo en lecturas de carácter voluntario. Por su parte, el docente dispone de múltiples vías de acceso a contenidos que clarifican y complementan la impartición de las asignaturas.

En el siglo XXI el perfil profesional del periodista es cada vez más transversal. Las universidades están adaptando sus propuestas curriculares a este nuevo escenario. Ya en 2004, en el 60 por ciento de las escuelas de periodismo norteamericanas se preparaba a los estudiantes para trabajar en la producción multiplataforma (Castañeda, Murphy y Hether, 2005: 57-70). El rol del docente universitario también se debe redefinir para caminar en la misma línea, incrementando sus funciones no sólo para habilitar nuevos espacios de debate y aprendizaje en entornos digitales, sino también para defender una organización flexible y creativa, aceptar un reciclaje continuado de conocimientos e incluso promocionar aquellos trabajos de los alumnos que estén dotados de calidad profesional y facilitar la captación de egresados por parte de las empresas.

\section{Referencias bibliográficas}

CARRATALÁ, Adolfo (2011): "Propuestas de aprendizaje cooperativo con alumnos de periodismo: interacción cara a cara y online", en VALLE MEJÍAS, María Elena del y CALVO, Elvira (coords.): Cambios en los modelos educativos en el EEES. Madrid, Visión Libros, pp. 21-40.

CASALS, María Jesús (2006): "La enseñanza del periodismo y las nuevas tecnologías de la información y de la comunicación". Estudios sobre el Mensaje Periodístico, $\mathrm{n}^{\circ}$. 12. Madrid, Servicio de Publicaciones de la Universidad Complutense, pp. 5970 . 
CASTAÑEDA, Laura, MURPHY, Sheila y HETHER, Heather J. (2005): “Teaching Print, Broadcast, and Online Journalism Concurrently: A Case Study Assessing a Convergence Curriculum". Journalism \& Mass Communication Educator, nº 60. Sage, pp. 57-70.

CUADRADO, Manuel F., RUIZ MOLINA, María Eugenia y COCA, Mercedes (2009): "Participación y rendimiento del estudiante universitario en un proyecto docente interdisciplinar, bilingüe y virtual". Revista de Educación, $\mathrm{n}^{\circ}$. 348. Madrid, Ministerio de Educación, Cultura y Deporte, pp. 505-518.

GARCÍA, Anna (2008): "Las redes sociales como herramientas para el aprendizaje cooperativo: una experiencia con Facebook". Periodismo, Comunicación y Sociedad, $\mathrm{n}^{\circ}$. 5. Universidad de Santiago de Chile, pp. 49-59.

GORDÓN PÉREZ, Mercedes (1991): La enseñanza del periodismo en el mundo occidental. Estudio histórico y comparado de tres escuelas. Tesis doctoral. Madrid, Universidad Complutense.

MACHILL, Marcel, BEILER, Markus \& SCHMUTZ, Jochen (2006): "The influence of video news releases on the topics reported in science journalism". Journalism Studies, $\mathrm{n}^{\circ} .7$ (6). Taylor \& Francis, pp. 869-888.

MCADAMS, Mindy (2005): Flash Journalism: How to Create Multimedia News Packages. Nueva York, Focal Press/Elsevier.

PALOMO, Bella (2008): "Propuesta de un modelo virtual para la asignatura Periodismo Interactivo y Creación de Medios Digitales". Anàlisi, nº. 36. Universidad Autónoma de Barcelona, pp. 183-195.

TEJEDOR, Santiago (2008): “Ciberperiodismo y universidad: diagnósticos y retos de la enseñanza del periodismo on-line". Anàlisi, n ${ }^{\circ}$. 36. Universidad Autónoma de Barcelona, pp. 25-39.

WINFIELD, Betty H. (2008): Journalism 1908: Birth of a Profession. Columbia, University of Missouri Press.

\section{María Bella PALOMO TORRES}

bellapalomo@uma.es

Facultad de Ciencias de la Comunicación - Universidad de Málaga

Departamento de Periodismo

Campus de Teatinos, s/n - 29071 Málaga

Licenciada y doctora en Periodismo por la Facultad de Ciencias de la Comunicación de la Universidad de Málaga (UMA), donde ingresó como asociada en 1998. Ha sido profesora visitante en las Universidades de Washington (2004), Rutgers (2005) y Salvador de Bahía (2009), y consultora en la UOC. Sus investigaciones se han centrado en el estudio de Internet, la adaptación del periodista al entorno digital, el diseño y la innovación educativa. Actualmente es profesora titular en el departamento de Periodismo de la UMA, coordinadora del grado e investigadora principal del proyecto nacional " $\mathrm{Au}$ diencias Activas y Periodismo: Estrategias de Innovación en la Empresa Informativa y Nuevas Figuras Profesionales". 